\title{
Expression visuelle de la structure des phrases en langue des signes française
}

\section{Visual Expressions Used to Represent Sentence Structures in French Sign Language}

\author{
Ertan KUŞÇU' ${ }^{10}$
}

${ }^{1}$ Assoc. Prof., Pamukkale University, Faculty of Science and Letters, Department of French Language and Literature, Denizli, Turkey

ORCID: E.K. 0000-0001-9945-9943

\section{Corresponding author: \\ Ertan KUŞÇU, \\ Pamukkale Üniversitesi, Fen-Edebiyat Fakültesi, Fransız Dili ve Edebiyatı, \\ Denizli, Türkiye \\ E-mail: ekuscu@pau.edu.tr}

Submitted: 21.07 .2020

Revision Requested: 24.07.2020

Last Revision Received: 30.09.2020

Accepted: 23.11 .2020

Citation: Kuscu, E. (2020). Expression visuelle de la structure des phrases en langue des signes française. Litera, 30(2), 779-801.

https://doi.org/10.26650/LITERA2020-0103

\section{RÉSUMÉ}

L'homme qui utilise les compétences de communication de la manière la plus compétente sur terre le fait en se référant soit au langage oral, soit à l'écrit ou au soit corporel. Cette dernière permet de transmettre l'information, l'émotion ou la pensée de la manière la plus correcte et efficace quand les mots sont insuffisants pendant la communication à chaque instant de la vie quotidienne. D'un côté, certaines réalités telles que la surdité et la perte auditive ont été les situations que les hommes rencontrent tout au long de l'histoire, donc, la langue des signes appelée parfois, la langue des sourds ou des sourds-muets, est utilisée par les sourds et les malentendants. D'autre côté, ces personnes isolées de la société jusqu'au XVIIle siècle en Europe, n'ont pas pu enrichir leurs langues et leurs vocabulaires, mais de nos jours, elles ont leur propre langue. Dans ce travail, tout d'abord, nous avons tenté de viser à expliquer la langue des signes française, ses périodes historiques et sa structure tout en recherchant théoriquement et en nous appuyant sur les ressources les plus récentes. Puis, sa structure syntaxique diffère du français oral, a été essayé d'expliquer par les dialogues courts accompagnés par les images dessinées. Ensuite, nous avons souligné la différence entre la structure de la langue des signes française (ordre libre ou OVS) et celle du français oral (SVO). Enfin, nous avons remarqué que les langues des signes, bien qu'elles soient des langues visiogestuelles, assurent toutes les fonctions remplies par les langues orales.

Mots-clés: Communication, langage corporel, langue des signes française, signe, signeur

\section{ABSTRACT}

An individual who proficiently uses communication skills does so by referring to either oral, written or bodily language, with the latter allowing information, emotions or thoughts to be conveyed in the most correct and effective way when words are insufficient. In general, individuals throughout history have faced deafness and hearing loss, resulting in the use of sign language to communicate with the outside world. This was apparent in Europe prior to the $18^{\text {th }}$ century, when such individuals were isolated and forced to create their own sign languages. Thus, the purpose of this study is three-fold: 1) to explain French sign language, its historical periods and structure, while theoretically researching and drawing on 
the latest resources; 2) to explain its syntactical and structural differences from oral French by examining short dialogs and drawn images; and 3) to highlight the differences between sentence structures in French sign language [e.g., free order or object-verb-subject (OVS)) and that of oral French (subject-verb-object (SVO)]. Based on the findings, although French sign language is visio-gestural, it can be effectively used to perform all functions in oral French.

Keywords: Communication, body language, French sign language, visio-gestural, signer

\section{EXTENDED ABSTRACT}

According to their origins, languages can be divided into two categories: natural languages (both spoken and sign languages) such as Turkish and French; and artificial languages such as Esperanto. In general, individuals who do not suffer from hearing loss generally use both spoken and body languages. However, for those who have a hearing loss, a visual form of communication also known as "sign language" or "deafmute language" is indispensable. In this regard, the current number of individuals with hearing loss is significant. Although a vast majority of them faced difficulties adapting to social life due to their lack of education, recent developments in the field of education have enabled them to acquire better education and improve their everyday lives. Additionally, with the formulation of various undergraduate and graduate programs, many sign languages studies have been published over the past few decades. In fact, there are 2,460 scientific publications (from 1990 to 2013) on sign languages in the Web of Science database (TID, 2005, p. 48).

In Europe until the $18^{\text {th }}$ century, individuals suffering from hearing loss were isolated from society, thus forcing them to create their own form of communication. In France, C. Michel de l'Épée was the first to work on French sign language (LSF or Langue des Signes Française), after discovering its existence. According to the widely known story, he witnessed twin deaf girls on a street in Paris communicating with one another by making a series of signs. Subsequently, he established a school for the deaf and developed a system of "methodical signs" to teach students how to read and write. After the French Revolution, this school became the National Institute of the Young Deaf.

Overall, the main elements of effective and correct communication in LSF are signs, just as words are for an oral language. These signs consist of five separate parameters: configuration, orientation, movement, location, and facial expressions. According to the context, they must be correctly performed to accurately signify the word; however, 
if the parameter changes, then another sign is used. Ultimately, facial expressions are included, just as intonations are used for those who can hear. Such expressions that accompany signs also provide information on the form of the sentence (i.e., interrogative, affirmative or question). Moreover, configurations, such as the shape or disposition of the hand, are equally important. In this regard, there are approximately 47 different configurations in LSF, such as the index, thumb, middle finger, etc. For example, in order to say "hello" in LSF, the hand leaves the mouth, while smiling.

As in all languages, LSF includes its own syntax and grammar. However, its syntax differs from that of oral French. In this regard, while French includes a subject-verbobject (SVO) structure, LSF responds in the following order: When? Where? Who? What? For example, in French we say, "The baby sleeps in his cradle," whereas in LSF, we sign "cradle, baby, sleep." In literature, this order resembles a theatrical scene in which the "curtain rises" and reveals the statement, after which the audience sees the setting, the characters, and the action. Additionally, in LSF, the sign for "where do you live?" includes two signs: "where," in which a lateral movement and an interrogative air is used; and "live," in which we open and close both hands simultaneously. Moreover, to say "I live in Paris," we make the sign " $\mathrm{P}$ " in the center of the hand to symbolize Paris. There are also no conjugations. Thus, to indicate the tense of a verb, we simply bring our hands closer or farther away. As for the past, we place the hands behind the body, whereas for the present and future, we bring the hands in front of the body. Finally, many verbs in LSF can become either multi or unidirectional, while the same sign can be used to indicate two meanings. For example, "campagne: campaign" and "province: province" are shown with the same sign. To summarize, LSF is a natural language that includes the same linguistic features as oral French. 


\section{Introduction}

La communication, dérivée du latin communicare, qui est l'un des besoins les plus fondamentaux et naturels des êtres humains, de façon générale, est définie comme un moyen d'exprimer des émotions et des pensées, de se comprendre, de transmettre des connaissances, etc. Dans ce contexte, la communication, berceau de nombreuses et différentes études, est une action primordiale permettant aux individus de pouvoir maintenir facilement leur vie. Entre autres, Paul Watzlawick précise «qu'il est impossible de ne pas communiquer » (Poirot, 2013, p. 32) à aucun moment de la vie; tout est communication.

Bien que la communication verbale est l'une de la plus utilisée du partage d'émotions, de pensées et de connaissances, le langage corporel en tant que les mouvements du visage, des mimiques est autant efficace que les mots et les phrases dans un processus de communication. A cet égard, Burgoon précise que «les comportements non verbaux, représentent environ 60 à $65 \%$ de la communication interpersonnelle et dans certains cas, peuvent constituer 100\% » (1994, p. 229-285).

Comme on le sait, « un bébé, avant d'apprendre à parler, utilise son langage corporel » (Baltaş, 2005, p. 11). Il communique avec sa mère en l'utilisant et dans sa prochaine vie, il continue à utiliser tantôt son langage corporel, tantôt son langage verbal. En effet, I'utilisation du langage corporel peut être plus pratique et plus efficace dans des actions comme " agiter les bras pour interpeller quelqu'un » (Marchal et Tessier, 2019, p. 4), hausser les épaules « en signe de doute, d'ignorance ou d'indifférence »', lever la main pour demander la parole ou parler et lever le pouce pour dire que tout va bien, etc. Mais tous " ces gestes et mimiques qu'on fait ne constitue pas une langue pour autant » (Marchal et Tessier, 2019, p. 4). Parce qu'une langue comme le français et le turc nous permet une communication complète, efficace, claire et riche avec un système d'unités bien organisées de manière précise.

Parfois, dans un processus de la communication, on utilise le corps et les mains plus que des mots. Car, les gestes, les mimiques, les silences, la posture, les expressions de visage, les signes et les manières de parler et d'établir un contact visuel et gestuel sont autant de moyens de communication non verbaux influençant les paroles et les messages

1 https://www.languefrancaise.net/Bob/42013, consulté le 05 mars 2020. 
que l'on diffuse verbalement. Comme on l'a souligné ci-dessous plus haut que tout est communication. Mais d'une part, il ne faut pas oublier que tous ces gestes que l'on fait ne forment pas seule une langue pour autant. Mais d'autre part, quand la perte d'audition et la surdité empêchent la communication, il est inévitable d'utiliser le langage corporel et aussi la langue des signes.

\section{La langue des signes française et la surdité}

Il existe de nombreuses définitions sur la langue des signes dans ce domaine. En général, la langue des signes (comme les mouvements des mains, du visage et du corps) que les sourds ont développée pour communiquer, remplit toutes les fonctions des langues orales. D'après Marchal et Tessier, lorsque des mots dans les langues comme le turc, le français, etc., sont produits oralement, dans les langues des signes, « ils sont réalisés à l'aide des gestes et des mouvements des mains. Ces derniers qui sont la base de la langue des signes sont ce que l'on appelle en général des signes » (2019, p. 4). D'après le dictionnaire Larousse, la langue des signes est « système structuré de gestes et d'expressions du visage conventionnels permettant aux sourds et aux malentendants d'exprimer et de communiquer leur pensée $»^{2}$. Sur la base des définitions, chaque langue des signes est une langue visio-gestuelle qui s'exprime avec des expressions du visage et des mouvements du corps.

La langue des signes française (désormais LSF), « appelée parfois, à tort, comme le langage des sourds-muets a été longtemps utilisée par une partie des sourds en France et aussi on l'appelle comme le langage de sourds-muets et des signes dans de nombreux ouvrages » (Marchal et Tessier, 2017, p. 3). Dans le domaine de la langue des signes, il est admis qu'elle est « née d'une série de signes créée par les moines du monastère pour faciliter les expressions et les rites religieux » (Caradec, 2005, p. 33). Mais ces personnes sourdes-muettes n'ont pas pu enrichir leur langue et leur vocabulaire car elles ont longtemps été isolées de la société.

D'après les estimations de L'OMS pour 2018, le nombre de personne ayant une déficience auditive augmente de plus en plus. Dans ce cadre, « il y a 466 millions de personnes dans le monde avec une déficience auditive $(6,1 \%$ de la population mondiale), 432 millions (93\%) d'entre eux sont des adultes (dont 242 millions d'hommes et dont

2 https://www.larousse.fr/dictionnaires/francais/signe/72700/locution?q=la+langue+des+signes\#11039520, consulté le 06 mars 2020. 
190 millions de femmes), 34 millions (7\%) d'entre eux sont des enfants. De plus, environ un tiers des personnes de plus de 65 ans souffrent d'une déficience auditive. D'autre part, à moins que certaines mesures ne soient pas prises, il est probable que le nombre de personnes ayant une déficience auditive augmentera au cours des prochaines années. Même, ce nombre pourrait atteindre 630 millions d'ici 2030 et dépasser 900 millions en $2050 »^{3}$. En plus, il y a environ « 3506800 personnes vivant en France qui souffrent actuellement de surdité $»^{4}$.

Aujourd'hui, la LSF est utilisée par « environ 150000 sourds et autant d'entendants en France. Mais ce nombre n'y représente qu'une petite partie de la communauté sourde » (Marchal et Tessier, 2017, p. 3). D'après les données datant de 2019 de la Fédération Nationale des Sourds de France, « un bébé sur 1000 est né comme sourd et dans la population, on estime à 300000 sourds, dont $1 / 3$ pratique couramment la langue des signes. Dont $34 \%$ est inactif du fait à la restriction d'accès à l'emploi, aux loisirs et à l'isolement $»^{5}$.

En 1998, selon une enquête faite sur les Handicaps-Incapacités-Dépendances (HID) par L'Union Nationale pour I'Insertion Sociale des Déficients Auditifs (UNISDA), « les difficultés d'audition concernaient plus de 5 millions de personnes en France, dont 300 000 souffraient d'une déficience auditive profonde ou totale et d'autres sont malentendants » (Laurence Haeusler, De Laval et Millot, 2014).

Comme Millet souligne que «la surdité n'est pas un choix, c'est un être, un mode de perception si l'on veut, qui fait tout de même que les sourds sont physiquement étrangers à la langue qui les environne. » $(1988$, p. 7$)$ ainsi pour des raisons d'ordre physiologique, « il leur est plus difficile que pour toute autre communauté linguistique d'apprendre la langue dominante » (De Pietro et Rispail, 2004, p. 146). Dans ce contexte, il faut que le son doive être perçu par le récepteur pour qu'une communication verbale puisse avoir lieu et aussi que ce son perçu par l'oreille humaine soit mesuré en décibels (dB) qui est l'unité de l'intensité sonore.

En France, comme indiqué ci-dessus, 5 millions de personnes souffrent d'un déficit auditif ou de la surdité qui sont liées à la perception des fréquences et des sons. D’après

3 https://www.who.int/deafness/estimates/en/, consulté le 07 mars 2020.

4 https://www.ethnologue.com/country/FR, consulté le 10 mars 2020.

5 https://www.fnsf.org/, consulté le 15 mars 2020. 
les chiffres fournis par l'Union Nationale pour l'Insertion Sociale de Déficients Auditifs (UNISDA), il existe « 4 degrés de surdité qui sont délimités par des seuils de perte auditive globale » (Marchal et Tessier, 2017, p. 3).

Perte auditive légère : C'est la perte de 21 à $40 \mathrm{~dB}$. «La parole est perçue à voix normale, difficilement perçue à voix basse ou lointaine. La plupart des bruits familiers sont perçus ${ }^{6}$. Cette déficience auditive légère concerne à peu près 2200000 personnes en France.

Perte auditive moyenne : c'est la perte se situe entre 41 et $70 \mathrm{~dB}$. La personne comprend mieux si elle regarde son interlocuteur ou les mouvements labiaux du locuteur. Ce degré concerne presque 1320000 personnes en France.

Perte auditive sévère : la perte se situe entre 71 et $90 \mathrm{~dB}$. La parole est pue percevoir quand on parle fortement près de l'oreille. Seuls les bruits forts sont perçus. Il s'agit de 360000 personnes atteintes de ce seuil en France.

Perte auditive profonde : C'est la perte est supérieure à $90 \mathrm{~dB}$. Il est impossible de percevoir la parole à ce seuil, on entend seulement les bruits très forts. C'est-à-dire la communication est impossible sans aide auditive. S'il s'agit d'une perte de $120 \mathrm{~dB}$ et plus, aucun bruit n'est perçu et la surdité est totale.

\section{Histoire de la langue des signes française}

L'histoire de la langue des signes est probablement aussi vieille que celle de I'Humanité. Comme expliquent Marchal et Tessier que " pour certains historiens et linguistes français, avant l'utilisation du langage oral, la première trace de la communication entre les hommes préhistoriques, se serait faite de manière visuelle et gestuelle » (2017, p. 4). Parce qu'il existait des écritures anciennes restées de grandes civilisations antiques comme " la Mésopotamie, l'Égypte, la Grèce, et inscrites sur des

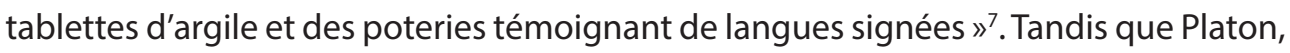
I'un des philosophes les plus connus de l'Antiquité, pensait que les sourdes avaient leurs propres langages, son disciple Aristote disait aussi que la seule chose différenciant I'Homme de l'animal était le langage. D'après lui, s'ils ne pouvaient pas parler, s'ils ne

6 http://www.wombatoak.com/?page_id=78, consulté le 18 mars 2020.

7 http://laboiteasaussure.fr/Isf_histoire.htm, consulté le 20 mars 2020 
pouvaient pas entendre, ils pouvaient être limités dans leur intelligence Donc, cette conception négative a longtemps duré dans l'esprit des gens.

Au Moyen Age, cette conception négative pour les sourds n'a pas beaucoup changé. Néanmoins, ils ont obtenu certains droits aussi dans cette période. Par exemple, lors de Concile d'Orange en 529, « ils ont eu le droit de demander le baptême par signes. Un an après, les sourds qui étaient privés de tous leurs droits civils avaient toutefois le droit de travailler notamment dans le champ » (Marchal \& Tessier, 2017, p. 4). Par contre, comme l'a dit Gök (1958), « les sourds qui semblent menacés, privés de compassion, ont été renvoyés loin de chez eux ou ont été tués par leurs familles » (citant Kaya, 2016, p. 166).

Dans les années 1000, grâce aux moines cisterciens, une langue des signes et un petit dictionnaire de gestes ont été créés afin qu'ils puissent faire leurs prières facilement. Cet effort était l'un des premiers pas les plus importants dans l'élaboration d'une langue signée commune. Puis Clairvaux, leur chef, a préparé « un dictionnaire dans lequel 227 signes se trouvent concernant à la vie monastique montrant les objets le plus utilisés comme les boissons, les ustensiles et les membres de la communauté, etc. » (Marchal \& Tessier, 2017, p.4).

Pendant l'Europe médiévale, il existait de nombreuses langues de signes utilisés dans les églises, et les deux d'entre-elles ont été formées au XI siècle. " L'une était la langue Uldaric qui avait le moins de signe avec 52 signes, l'autre était la langue de signes formées par Guillaume d'Hırsan qui avait le plus de 365 signes » (Caradec, 2005, p. 32). Au XII siècle, le pape III a décidé qu'un(e) sourde(e) et un(e) entendant(e) « se marieraient à condition qu'un(e) puisse dire « oui » par le signe. Mais d'autre part, il était encore interdit du mariage entre sourds » (Marchal \& Tessier, 2017, p. 4).

Dès la Renaissance, les sourds commencent peu à peu à profiter des activités scolaires. Au XVlème siècle, le moine Pedro Ponce de León, commençant à s'intéresser « aux enfants sourds, issus de la noblesse espagnole et à leur éducation ouvre une classe spécialisée dans son monastère » (Marchal \& Tessier, 2017, p. 4). Pour eux, De León créant un alphabet qui permet d'épeler un mot essaie de leur apprendre comment s'exprimer par des gestes très simples. Donc, c'est la naissance de l'alphabet de la dactylologie. Par ordre, « En 1620, Juan Pablo de Bonnet publie le premier ouvrage consacré à l'éducation des enfants Sourds. Un siècle plus tard, le précepteur Jacob 
Rodrigues Pereire est le premier à éduquer des enfants sourds issus de la noblesse française $»^{8}$.

En 1760, l'abbé Charles Michel de l'Epée qui « considère comme l'une des figures les plus importantes pour les sourds en France est le premier s'intéressant à la communication gestuelle des sourds » (Marchal et Tessier, 2017, p. 4). Selon la légende la plus répandue, alors que L'abbé de l'Epée se promène dans les rues de Paris, il observe à travers une fenêtre embuée un couple de jumelles sourdes communiquant d'entre elles par leurs gestes. Etant fasciné par leur manière de communiquer, « il décide de s'appuyer sur cette langue pour instruire les enfants sourds en adaptant et en ajoutant des notions grammaticales propres au français (par exemple, la conjugaison). C'est ce qu'il appelle les signes méthodistes $»^{9}$. Après cet événement, d'abord, L'abbé de l'Epée ouvre la première école gratuite pour les sourds et les malentendants, et puis écrit son propre dictionnaire en langue de signes en utilisant un signe pour chaque mot. Plus tard, un jour « il y invite le roi Louis XVI afin de montrer comment les jeunes sourds sont capables d'écrire leur nom en épelant grâce à la dactylologie » (Marchal et Tessier, 2019, p. 4).

Après la Révolution Française, l'éducation des sourds est considérée comme une affaire nationale et l'école précédemment fondée par l'abbé de l'Epée, devient l'Institut National des jeunes Sourds (INJS). Après sa mort, alors que les sourds sont plus adoptés et plus embrassés par la société qu'auparavant, eux aussi pour mieux communiquer, commencent à utiliser leur mimique et les signes méthodiques. Comme Marchal et Tessier citent qu'en 1817, Thomas Hopkins Gallaudet et Laurent Clerc « fondent une autre école pour les enfants sourds. Clerc adapte la LSF à la langue anglaise. Grace aux efforts de Gallaudet, l'ensemble des cours dans cette école est donnée en langue des signes » $(2017$, p. 6). En plus, Ferdinand Berthier est également connu comme le premier sourd obtenant le titre du professeur en 1829.

Mais, cet âge d'or de la LSF qui dure depuis des centaines d'années, se termine par une décision prise au congrès de Milan en 1880. A la fin du congrès, " une méthode orale qui porte sur la labiolecture et la production de mots pour éduquer les sourds est choisie » (Marchal et Tessier, 2017, p. 6). Par conséquent, il était proposé d'attacher leurs mains dans le dos pour qu'ils empêchent de communiquer en utilisant la LSF.

8 http://laboiteasaussure.fr/lsf_histoire.htm, consulté le 23 mars 2020.

9 https:// fr.wikipedia.org/wiki/Langue_des_signes_française, consulté le 23 mars 2020. 
Cette pratique a duré plus de cent ans. Mais malgré cette interdiction, les sourds français continuent à pratiquer leur langue.

De nos jours, on se voit que les personnes sourdes et malentendantes qui pratiquent la langue de signes semblent réussir dans de nombreux domaines comme les entendants. Par exemple, bien que « Hellen Keller ne puisse pas parler, entendre et voir, elle est également connue pour ses succès dans le domaine de la pédagogie et comme la reine des aveugles, des sourds et des muets; on sait que Beethoven interprète la $9^{\mathrm{e}}$ symphonie pendant sa période de surdité » (Kaya, 2006, p. 166).

Selon une loi publiée en 1975, les enfants sourds en France sont fixés recevoir une éducation obligatoire, un an après, Jean Grémion et Alfredo Corrado décident de créer un théâtre à Paris pour les sourds. Juste après, « une association d'interprétés en langue des signes française est créé. En 1991, grâce à la loi Fabius, la famille des enfants sourds permet de choisir entre la méthode oraliste et la LSF dans l'éducation de leurs enfants » (Marchal \& Tessier, 2017, p. 5 ; Marchal \& Tessier, 2019, p. 7).

Les langues des signes sur lesquelles il existe de nombreuses études expérimentales sont également utilisées dans de nombreux pays du monde. L'une d'elles a été faite sur les enfants sourds dans une école spécialisée à Mangua, la capitale du Nicaragua, au début des années 1980. D’après cela ;

La première génération de jeunes sourds scolarisés dans l'établissement ne maîtrisait pas la langue des signes : leurs familles ne « signaient » pas et le personnel de l'école non plus, car leur objectif était de les préparer à l'oralisation et à lire sur les lèvres. Au fil des mois, les élèves ont spontanément mis au point un code gestuel pour communiquer entre eux. Mais ce n'était pas un vrai langage, plutôt une sorte de pidgin, de sabir suffisant pour se dire «toi jouer avec moi dans la cour » mais inapte à remplir toutes les fonctions du langage, à accéder à l'abstraction, à émanciper totalement la communication de l'instant, du seul présent des sensations. La surprise est venue avec la deuxième génération d'enfants entrée à l'école : tout « naturellement ", ces jeunes sourds ont transformé cette communication gestuelle en langage des signes doté d'une grammaire, d'une syntaxe...etc. Bref, en véritable langue capable d'exprimer toute la richesse et la complexité de la pensée humaine (Lestienne, 2008, p. 8). 
Dans l'analyse des ressources, il est clair que la langue des signes a été enseignée non seulement aux sourds mais aussi aux animaux au XVIIle siècle. Par exemple, Lord Mondobbo au Royaume-Uni prétend qu'on pourrait apprendre à parler à un singe pourvu qu'on le prenne assez jeune. Dans les années 1970, Allen et Beatrix Gardner a élevé « une petite femelle chimpanzé, Washoe, comme un garçon sourd et ont essayé de lui apprendre la langue des signes américaine. Mais tous ces efforts sur les animaux ont échoué » (Lestienne, 2008, p. 8).

Le linguiste américain Steven Pinker précise que «la langue est un instinct génétiquement programmé et à l'exception de quelques graves pathologies, tout le monde peut parler, y compris les sourds parlant en langue des signes » (Lestienne, 2008 , p. 4). Comme on l'a expliqué, il existe de nombreuses façons de communiquer. L'une d'elles es la langue des signes qui est une langue visuelle et gestuelle s'exprimant uniquement avec des expressions du visage et du corps, utilisée par les sourds, les personnes malentendantes et leurs familles n'est que l'une d'entre elles.

\section{Les caractéristiques de la langue des signes française}

Pour pouvoir communiquer, le principal élément de toutes les langues des signes est le signe, comme les mots pour une langue orale. D'après Marchal et Tessier, « ces signes se composant de divers paramètres, peuvent être expliqués et imités » (2017, p. 8 ; 2019, p. 5). Chaque signe peut être décrit en cinq paramètres corporels. Si un paramètre change, on obtient un autre signe. Ces paramètres sont, par ordre, la forme de la main ou la configuration, l'orientation, l'emplacement, le mouvement et l'expression du visage. Par exemple pour les sourds américains, ces expressions du visage pour les sourds faisant partie intégrante de la langue des signes sont comme l'intonation d'entendants.

La configuration, autrement dit la forme de la main ou les dispositions de la main. En langue des signes française, il existe « environ 47 configurations différentes à savoir comme l'index, le pouce, le majeur, l'auriculaire, la main ouverte, la main plate, la main creuse, le poing etc. » (Companys et Tourmez, 2015, p. 5). Pour dire « bonjour » dans la LSF (Tableau 1), la main part de la bouche en souriant. 


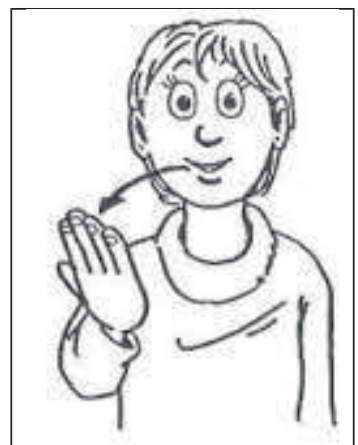

Tableau 1. «Bonjour » en langue des signes française (Companys et Tourmez, 2015, p. 14).

Le mouvement, accompagne le signe. En langue des signes française, il n'y a pas de signe sans mouvement et tous les signes nécessitent de bouger les bras. Il est difficile de « transcrire ces mouvements sur les pages du dictionnaire et à interpréter, car les mouvements sont en trois dimensions mais les dessins ne sont pas en relief » (Companys et Tourmez, 2015, p. 10). Par exemple, l'affichage des mots « clé, ça, venir, vélo » en langue des signes française sont comme le suivant (Tableau 2).
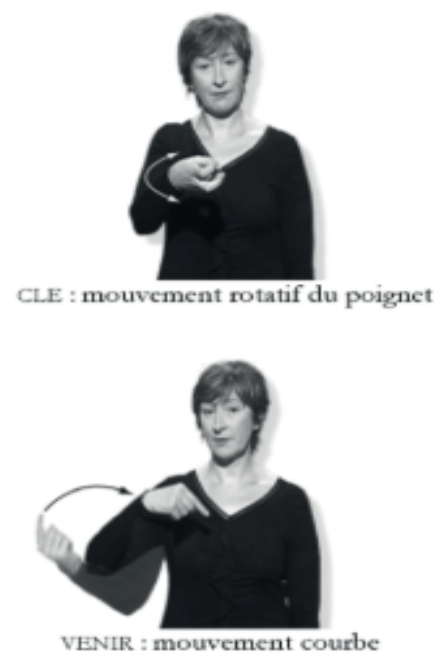
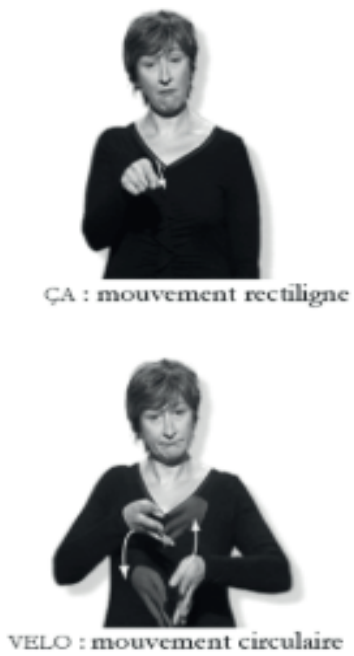

Tableau 3. Les signes « clé, ça, venir, vélo » en langue des signes française (Dabin, Verhulst, 2009, p. 40)

Le mouvement se fait généralement vers soi, vers l'avant, de gauche à droite, en spirale, du haut vers le bas, dans le sens circulaire, d'ouverture, de fermeture et de contact. Ils sont simples, complexes ou répétés. 
L'orientation qui est la position de la paume de la main par rapport au signeur se réalise vers le haut, le bas, l'extérieure, la gauche, la droite, I'arrière, vers soi. La paume peut être orientée comme la suivante (Tableau 4).

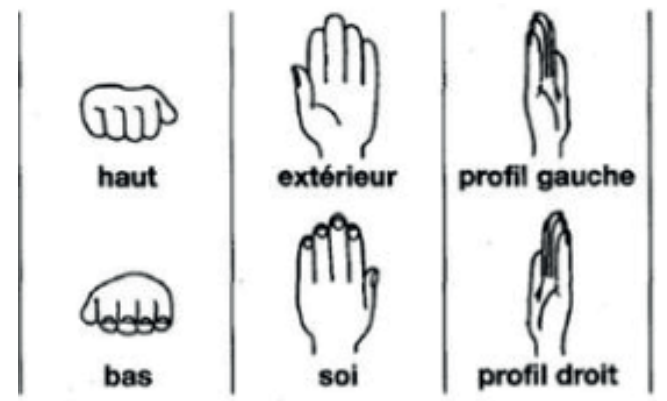

Tableau 4. La paume orientée (Companys et Tourmez, 2015, p. 9).

Pour signer le mot « ordinateur » (Tableau 5) en langue des signes française, sa configuration, son emplacement, sont orientation sont comme ci-dessus.

\section{ORDINATEUR}

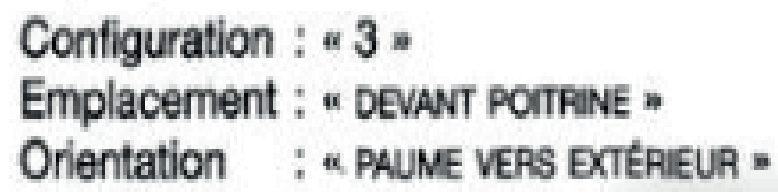

Tableau 5. «Ordinateur » en langue des signes française.

L'emplacement d'un signe concernant la personne qui signe. C'est-à-dire, « la place du signe par rapport au signeur » (Companys et Tourmez, 2015, p. 8) peut apporter une information sur le sens. Dans l'espace, on distingue quelques endroits principaux. Si un signe est au-dessus de la tête, c'est l'imaginaire ; s'il est au niveau du front, c'est la réflexion ; " s'il est au niveau de la poitrine, c'est les sentiments ; s'il est au niveau de l'estomac, c'est l'alimentation » (Marchal \& Tessier, 2019, p. 5). Il existe plus de 30 zones totales sur notre corps où se font les signes LSF. Parfois, un emplacement qui n'a pas de contact physique direct (Tableau 6) en ne touchant pas le signeur; parfois un emplacement précis du signeur plus un contact réel (Tableau 7) en touchant le signeur. 


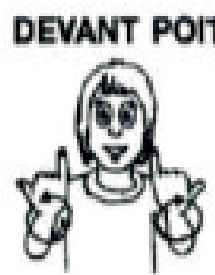

JOUER

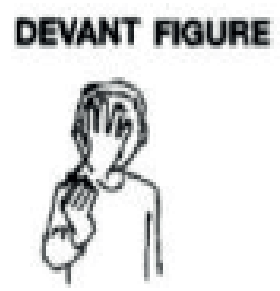

S'ENDORMIR
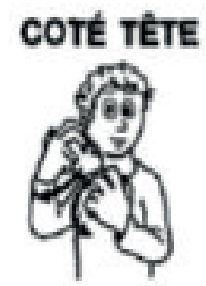

BRUIT

Tableau 6. Des signes devant la poitrine, le visage, sur le côté de la tête

(Companys et Tourmez, 2015, p. 8).

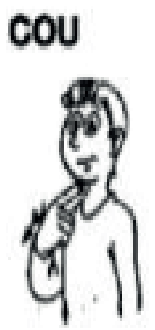

ALCOOL
NEZ

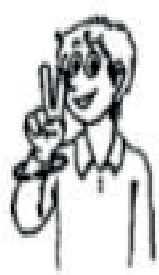

COMIQUE
FRONT

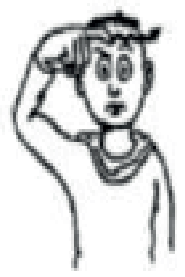

DIFFICILE

Tableau 7. Quelques points précis délimités en langue des signes française

(Companys et Tourmez, 2015, p. 8).

L'expression du visage ou corporelle qui « donne des informations temporelles, qualitatives et quantitatives » (Marchal et Tessier, 2019, p. 5). Tous les entendants, les malentendants et les sourds utilisent les expressions du visage tous les jours. Car cela fait partie de la façon de communiquer. En plus, ces éléments grammaticaux en langues des signes française se retrouvent sur le visage. Dans ce contexte, ces expressions du visage sont aux sourds ce que l'intonation est aux entendants. Comme Marchal et Tessier précise qu'« on ne peut pas avoir un visage impassible » (2015, p. 11). Quand on voit un sourd, ses yeux, ses sourcils, sa bouche et son visage etc. sont foncièrement parlantes. 


\section{La structure des phrases en LSF}

De même que dans toutes les langues parlées et les langues des signes du monde, la LSF possède son propre ordre des mots, sa propre grammaire etc. Mais son ordre des mots est donc différent de celui du français parlé. C'est-à-dire, tandis que ce dernier s'appuie sur la structure « sujet + verbe + complément », en tant que le langage visuel et gestuel, une phrase en LSF répond à un ordre bien précis comme « quand ? où ? qui ? quoi ? ». Par exemple, on dira ainsi en français parlé, « le bébé dort dans son berceau ». Mais on signera en LSF dans cet ordre « berceau, bébé, dort ». Pour un sourd, cette langue peut être très facile, mais pour un entendant, un peu compliquée parce qu'il faut lui signer à l'envers du français parlé. Cet ordre des mots est comme une scène de théâtre, on voit d'abord le décor, puis les acteurs entrant en scène, enfin l'action commence.

En français oral, il est obligatoire de conjuguer le verbe en genre et en nombre. Pourtant la LSF ne possède pas de conjugaison et on modifie «le temps des verbes en éloignant ou rapprochant les mains de soi » (Picq \& autres, 2008, p. 6).

Pour poser une question en LSF, les mots interrogatifs tels que « que, quoi, où, comment etc. » sont signées en fin de phrase. Par exemple, on dira ainsi, en français, « Vous mangez au restaurant ?» on signera en LSF dans cet ordre « restaurant, vous, manger?».

Il est difficile de communiquer pour tous ceux qui ne connaissent pas l'ordre des mots en LSF. Par exemple, quand on dit en français « Je regarde ces oiseaux »; cela se range syntaxiquement « oiseaux ces regarder » en LSF. De plus, il est possible d'exprimer simultanément beaucoup d'idées en tant que celles du français oral. Par exemple, lorsqu'on dit : «Aujourd'hui, j'ai visité la tour Eiffel », on signe d'abord « aujourd'hui », ensuite «la tour Eiffel » et enfin « visiter » en profitant des expressions du visage et des gestes. Pour un sourd, la grammaire de la LSF est plus facile que celle du français mais, pour entendant, c'est l'opposé. D'autre part, afin de comprendre ce qu'on dit et de parler facilement la LSF, il convient de rappeler qu'il est nécessaire de recourir aux expressions du visage et d'autres paramètres mentionnés ci-dessus.

En LSF, il existe deux types de verbes tels que les verbes pluri-directionnels et unidirectionnels. Si un verbe prend deux pronoms, il est appelé le verbe pluri-directionnel. 
Par exemple, le locuteur, pour signer " Je vous téléphone », fait d'abord le signe " téléphone » de lui vers son interlocuteur. Et puis, son interlocuteur, pour lui répondre, «Vous me téléphonez », se fait d'abord en signant « téléphone » de lui vers son locuteur. S'il s'agit d'un verbe qui prend un pronom, cela s'appelle un verbe uni-directionnel, de cette manière, le signeur utilise un seul pronom comme " je mange », « il va » et « tu travailles » etc.

Il existe de différentes opinions sur l'ordre des mots en LSF qui fait toujours l'objet des nombreuses études. Tandis que « certains la considèrent comme une langue d'ordre libre, d'autres l'acceptent l'ordre syntaxique OVS (objet, sujet, verbe). En conclusion, l'ordre des mots en LSF est comme le suivant: d'abord le lieu, puis le temps, ensuite le sujet et enfin l'action. Cet ordre est plus concrètement comme une mise en scène : le décor est tout d'abord planté, les acteurs entrent ensuite en scène et l'action peut enfin débuter $»^{10}$.

Lorsque l'on fait connaissance, il peut être important de se renseigner pour savoir si on est sourd ou entendant. Donc, en langue des signes française comment est-ce qu'on peut le demander? En français, pour demander à quelqu'un(e) s'il est sourd ou entendant ;

Mireille : Est-ce que tu es sourd ? (Sourd, toi), (Tableau 8).

Pierre : Non, je suis entendant. (Non, entendant), (Tableau 9).

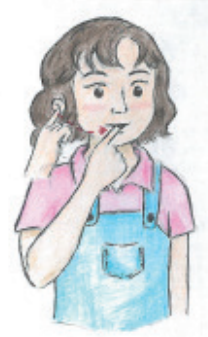

Tableau 8. Sourd, toi ?
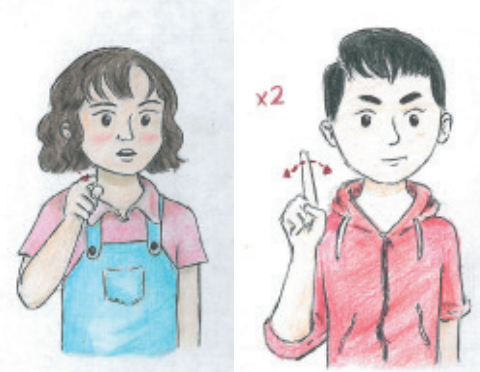

Tableau 9. Non, entendant.

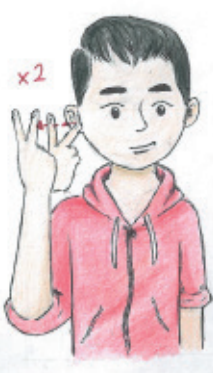

Pour commencer, Mireille signe de l'oreille à la bouche pour le signe «sourd » puis, en pointant avec son doigt vers Pierre à qui elle parle. Après cela, pour signer « toi »

10 https://sites.google.com/ site/languesignes/grammaire, consulté le 23 mars 2020. 
elle pointe l'index devant soi avec une mimique faciale jouant un grand rôle lorsqu'une question est posée. Ensuite, Pierre fait le signe «non » en agitant son index à gauche et à droite. Enfin, pour signer « entendant » il fait un mouvement sur l'oreille, deux fois, vers l'arrière. Pendant leur conversation LSF, tous les deux utilisent certains paramètres indispensables pour la formation des signes comme la configuration, l'orientation, l'emplacement, le mouvement et les expressions du visage.

Dans le tableau 8, l'ordre des mots qui n'est pas celui de la langue française est parfois considéré comme une langue d'ordre libre. Dans le tableau 8, l'adjectif « sourd » vient avant tout, puis le pronom « toi » vient. Dans le tableau 9, l'adverbe « non » et puis l'adjectif « entendant » viennent par ordre. Cela montre qu'il n'existe pas un ordre comme le sujet, le verbe et le complément en LSF. A la fois, en LSF les noms n'ont pas de genre comme féminin ou masculin. Dans la revue de littérature, on voit que l'ordre des mots en LSF est un sujet de recherche. En français et en LSF, pour demander à quelqu'un(e) si on sait signer :

Mireille : Sais-tu signer ? (Signer, savoir), (Tableau 10).

Pierre : Oui, je signe un peu ! (Oui, signer un peu), (Tableau 11).

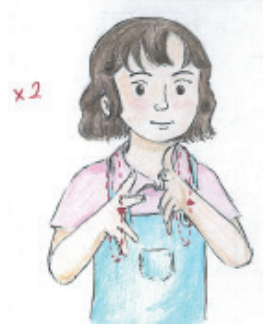

Tableau 10. Signer, savoir?
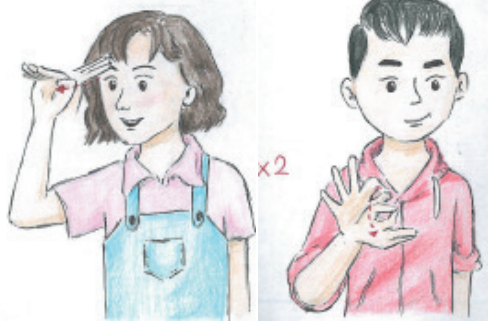

Tableau 11. Oui, signer un peu.

Il est sûr que le verbe « signer » est le premier signe à connaitre en LSF. Dans le tableau 10, Mireille fait deux fois le signe « signer » et ensuite elle signe une fois le verbe " savoir », au niveau du front, sur la tempe vers l'avant à l'aide de ses expressions du visage. Dans le tableau 11, Pierre signe « oui » deux fois à l'aide de ses doigts, avec son pouce et son index formant le « $\mathrm{O}$ », son majeur et son annulaire le « $U$ » et son auriculaire le « $\mid \mathrm{l}$ ». Enfin il fait le signe « entendant » avec un mouvement sur l'oreille, deux fois vers l'arrière.

Pierre et Mireille signent où ils vivent. En LSF, chaque ville ou chaque pays ont un nom-signé, comme les personnalités historiques, les sportifs, les artistes, les personnages 
de fiction et les marques etc. D’après la LSF, on se renseigne sur le lieu d'habitation comme le suivant :

Mireille : Où habites-tu ? (Habiter, où), (Tableau 12).

Pierre : J'habite à Paris, c'est une grande ville. Et toi ? (Paris, habiter), (Tableau 13) ; (Ville, grande, toi ?), (Tableau 14).

Pierre : A la campagne. (Campagne), (Tableau 15).

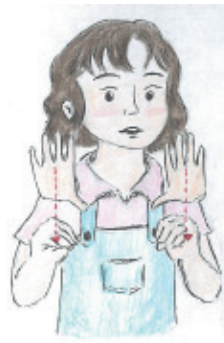

Tableau 12. Habiter, où?

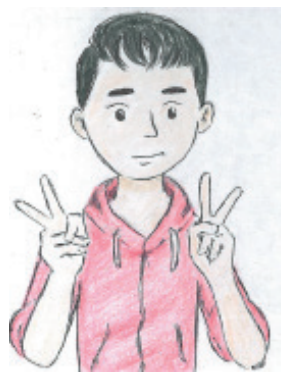

Tableau 14. Ville, grande, toi ?

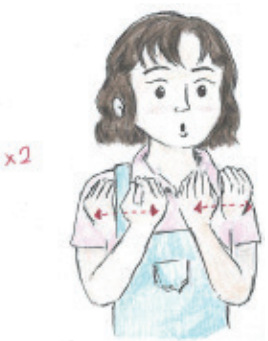

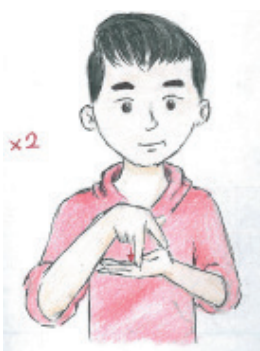

Tableau 13. Paris, habiter.

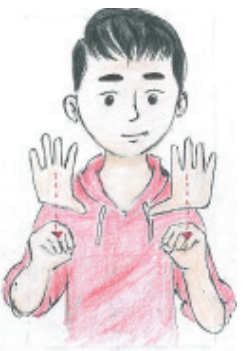

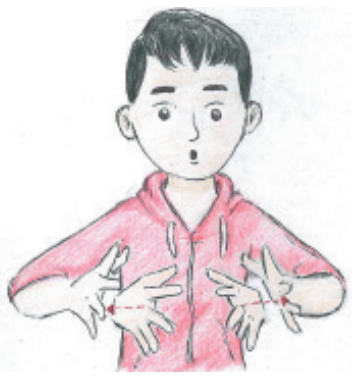

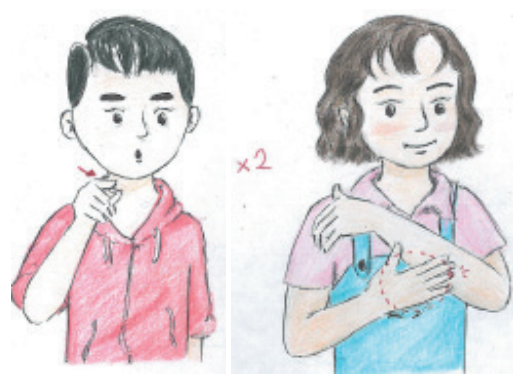

Tableau 15. Campagne

Dans le tableau 12, pour le signe « habiter», Mireille ouvre et ferme ses deux mains en même temps, une fois, sur place et pour « où », elle signe deux fois un mouvement latéral et un air interrogateur. En LSF, un nom d'une ville ou d'un pays portent sur des éléments emblématiques. Dans le tableau 13, Pierre fait la lettre de dactylologie « $P$ » pour signer Paris, c'est-à-dire, c'est la lettre « $P$ » signée au centre de la main qui symbolise Paris. Dans le tableau 14, pour le signe « ville », Pierre utilise la lettre de l'alphabet de la langue des signes française et alors ses paumes sont vers le haut, puis le bas, mais une fois. Pour signer " grande », Il ouvre ses mains au niveau de son ventre et les élargit en soutenant ses expressions du visage. Pour le pronom « toi », il pointe l'index devant soi et fait appel à sa mimique faciale. Dans le tableau 15, Mireille fait un mouvement 
circulaire sur le bras juste avant le coude pour le signe « campagne ». Ce signe a deux sens différents. «L'un est la campagne et l'autre est le province » (Marchal \& Tessier, 2019, p. 23). Pour demander l'âge d'une personne en LSF :

Mireille :Tu as quel âge ? (âge, toi ?), Tableau 16.

Pierre : J'ai 14 ans. Et toi ? (14, ans, toi ?), Tableau 17.

Mireille : J'ai 13 ans. (13, ans), Tableau 18.
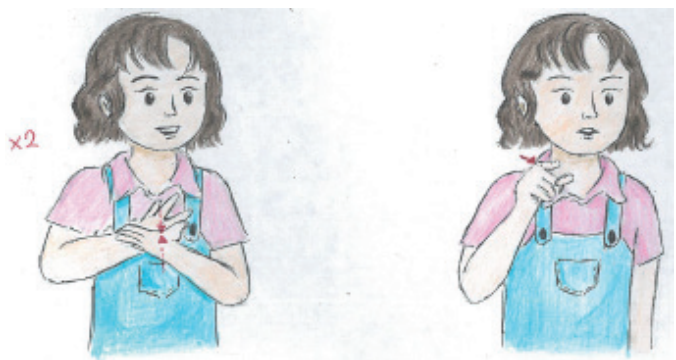

Tableau 16. Age, toi?
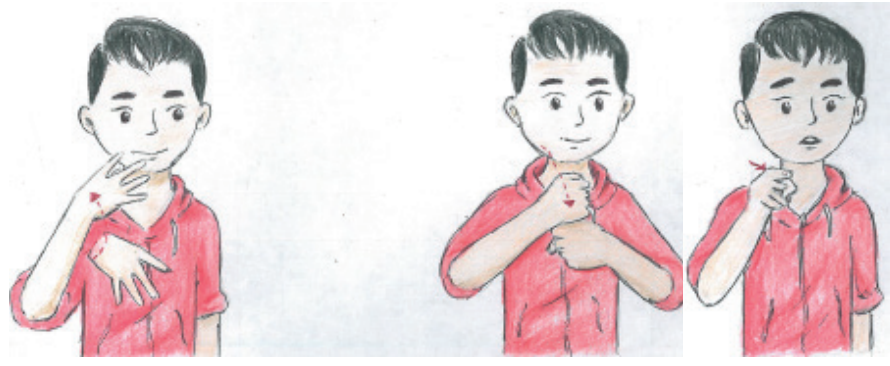

Tableau 17. 14, ans, toi ?

II n'existe pas d'une façon particulière de signer l'âge. II suffit de savoir juste comment signer les nombres. Dans le tableau 16, l'ordre des mots est comme le nom «âge » et le pronom « toi ? ». Le signe « âge » est fait en tapant les mains à l'horizontale, c'est comme les applaudissements. Dans le tableau 17, on utilise les doigts pour montrer les nombres et, des signes au-delà de 9 ont été adaptés de nombres entre 1 et 9 . D'après Companys et Tourmez, « les nombres 22, 33, 44, 55, 66, 77, 88, 99 se font par déplacement latéral » (2015, p. 15). Mais pour les nombres restants, par exemple 48 , on signe d'abord le chiffre 4 , puis 8 , non pas 40 et puis 8 comme le langage parlé. Pour signer « 14 » et « ans », Pierre fait d'abord le signe « 14 » et puis un poing immobile et l'autre fait demi-tour devant la poitrine. Après cela, pour le signe « toi ?», il pointe l'index devant soi afin de donner l'impression qu'une question a été posée à partir de sa mimique foncièrement faciale. 


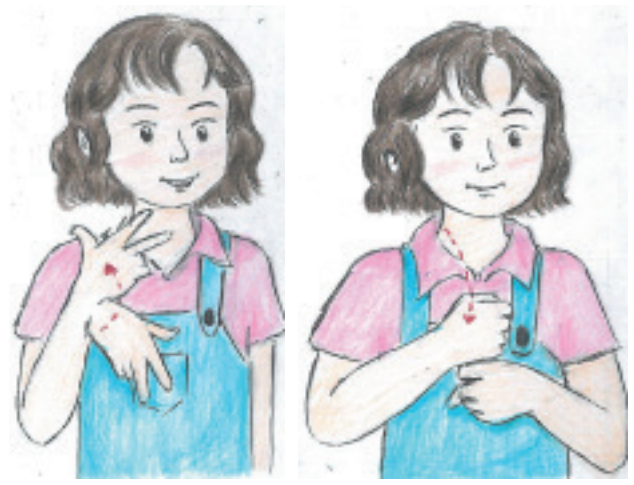

Tableau 18. 13, ans.

Dans le tableau 18, Mireille fait tout d'abord le signe « 13 » et juste après, signe « ans » avec ses deux mains, c'est-à-dire, un poing immobile et l'autre fait demi-tour devant la poitrine.

Comme indiqué précédemment, l'ordre des mots est donc différent entre la langue des signes française et le français oral. Par exemple, l'ordre des mots dans cette phrase « J'habite à Paris » est comme le sujet, le verbe et le complément. Mais cet ordre en LSF est comme le lieu et le verbe. Dans ces phrases étudiées, on voit qu'il n'existe pas de conjugaison. Pour indiquer le temps d'un verbe, il suffit de rapprocher ou éloigner la main. Pour le passé, on met les mains derrière le corps, pour le présent et le futur devant lui. En outre, pour mieux indiquer le sens d'une phrase ou d'un mot, on recourt aux expressions du visage.

La phrase en LSF répond à un ordre précis de questions : « où, qui, quoi ? ». Marchal et Tessier expliquent cela comme le suivant : « il s'agit, comme au théâtre quand le rideau se lève, de donner à voir l'énoncé. Tout d'abord, le spectateur aperçoit le décor, le lieu, où se passe l'action (où ?) ; ensuite le ou les personnages entrent en scène (qui ?) ; enfin ils se mettent en action (quoi ?) » (2019, p. 7).

En français oral, on fait une petite pause entre les mots prononcés dans une phrase, mais en LSF, la pause est faite entre chaque phrase, c'est à ne pas s'arrêter entre chaque signe. Comme déjà mentionné, les expressions du visage sont très importantes, car les yeux grands ouverts ou fermés, les épaules, les sourcils, etc. peuvent informer si la phrase est affirmative, négative ou interrogative en donnant des informations à l'interlocuteur sur le type de la phrase. 
Par conséquent, à partir des exemples et des interprétations ci-dessus, et aussi comme l'a dit Aydın que «les langues des signes ont certaines caractéristiques grammaticales et lexicales en commun, ainsi que certaines caractéristiques linguistiques communes à diverses langues parlées, mais il semble que les langues des signes diffèrent d'une communauté sourde à l'autre, de plus, de la même région d'un pays, tout comme les langues parlées » (Aydın, 2013, p. 79).

\section{Conclusion}

Lors d'une conversation orale, on profite généralement des mouvements du corps, des expressions de visage et des mains plus que des mots. Ce langage corporel non verbal fournit une communication plus efficace et soutient des paroles qu'on diffuse verbalement.

La langue des signes, appelée parfois la langue des sourds est un système linguistique possédant sa propre grammaire et syntaxe comme toutes les langues orales. Elle permet de communiquer, de transmettre des idées, des émotions et des connaissances. D’un autre côté, la langue des signes n'est pas une langue internationale et chaque pays a sa propre langue des signes. Mais, certains signes sont aisément compréhensibles tels que « je t'aime, boire, manger, etc. » dans toutes les langues des signes. A part, il existe des signes expliquant une phrase entière en un seul signe dans toutes les langues de signes.

La langue des signes française permet de tout dire comme les langues orales. Par ailleurs, l'utilisation d'un seul signe peut dénommer les scientifiques, les écrivains, les artistes et les personnages les plus connus, les villes, les pays, etc. S'il n'existe pas d'un nom signé on utilise la dactylologie qui sert à épeler tous les noms dont on ne connait pas le signe. De même que dans toutes les langues, en LSF, il faut respecter un ordre bien précis pour former des phrases quand on s'exprime. Pour commencer, il n'existe pas de temps du verbe, le positionnement des signes par rapport au corps donne aussi l'idée du temps (passé, présent, futur). L'ordre des signes en LSF est différent de l'ordre des mots du français oral. En général, le verbe se met en fin de phrase. Tout d'abord, on met toujours les compléments circonstanciels de temps, de lieu. Puis le sujet et ensuite l'action. A savoir, la phrase en LSF répond cet ordre de questions : « quand, où, qui, quoi ? ». Lorsqu'il s'agit de la négation dans une phrase, on la met après le verbe, en fin de phrase. 
Pour signer en LSF, on se sert de signes composés de paramètres que l'on peut distinguer séparément. Ces paramètres sont respectivement la configuration, l'orientation, l'emplacement, le mouvement et l'expression du visage. Si l'un de ces paramètres change, on obtient un autre signe. Alors tous ces paramètres entrent en jeu correctement pour communiquer en LSF.

En conclusion, il existe de deux conclusions tirées de cette recherche, l'une ; quoi qu'il en soit, il est impossible de ne pas communiquer. L'autre ; la base théorique de cette recherche est constituée par l'analyse de la structure des phrases de la langue des signes françaises. Tout d'abord, nous avons analysé la littérature sur ce sujet. Ensuite, nous avons sélectionné des phrases dans la langue parlée, celles-ci ont été accompagnées d'images représentant la syntaxe de la langue des signes. A la fin de cette recherche, nous avons remarqué qu'il y avait de grandes différences entre la syntaxe de langue des signes françaises et du français oral. Tout compte fait, quoi qu'il en soit, la communication orale ou corporelle est primordiale et indispensable pour chaque être humain.

Évaluation : Évaluation anonyme par des pairs extérieurs.

Conflit d'intérêts : L'auteur n'a aucun conflit d'intérêts à déclarer.

Subvention : L'auteur n'a reçu aucun soutien financier pour ce travail.

Peer-review: Externally peer-reviewed.

Conflict of Interest: The author has no conflict of interest to declare.

Grant Support: The author declared that this study has received no financial support.

\section{Bibliographie}

Aydın, A., Z. (2013). Sign languages and aspects of Turkish Sign Language (TiD). Hacettepe Üniversitesi Edebiyat Fakültesi Dergisi, 30 (1), 0-0. Consulté le 30 Septembre 2020, disponible sur https://dergipark.org.tr/tr/pub/ huefd/issue/41216/501954

Burgoon J.K, Buller, D.B, Woodall W.G. (1994). Nonverbal Communication: The Unspoken Dialogue, Greyden Press, Dayton, Ohio.

Caradec, F. (2005). Beden Dili Sözlüğü, (Çev. Ceyda Aktaş), 1. Basım, Kitap yayınevi Ltd., İstanbul.

Companys, M. (2015). Dictionnaire 1200 Signes, la Langue des Signes Française, Editions Monica Companys,Paris.

Dabin, I., Verhulst F. (2009). Création de comptines ou de chansons en langue des signes, Dans les coulisses d'un enseignement bilingue (langue des signes-français) à Namur, Le groupe de féflexion sur la LSFB, (Eds.) Laurence Meurant, Marie ZEGERS DE BEYL, Presses Universitaires de Namur. 
De Pietro, Jean-François \& Rispail, Marielle (dirs). (2014). L'enseignement du français à l'heure du plurilinguisme : vers une didactique contextualisée. Namur : Presses universitaires de Namur. (Recherches en didactique du français 6). 339 p. ISBN 978-2-87037-851-9.

Dikyuva, H., Makaroğlu, B., Arık, E. (2015). Türk İşaret Dili, Dilbilgisi Kitabı, T.C. Aile ve Sosyal Politikalar Bakanlığı, Impetus Mesya Prodüksiyon Ltd. Şti. Ankara.

Haeusleur, L. De Laval, T., Millot, C., (2014) Direction de la recherche, des études, de l'évaluation et des statistiques DREES, document de travail Série Etudes et Recherches No 131, Étude quantitative sur le handicap auditif à partir de l'enquête « Handicap-Santé ».

Kaya, Ç. (2009). Işsitme Engelliler ve Dil, Türk Dili Araştırmaları Yıllığı - Belleten, 54 (2006/1), 165-176.

Lestienne, C., Piq, P., Sagart, L., Dehaene, G. (2012). La plus belle histoire du langage, Editions du Seuil, Paris.

Marchal, O., Tessier, T. (2017). La langue des signes française, Editions Garnieret Le Monde.

Marchal, O., Tessier, T. (2019). Petit Guide de Conversation, en Langue des Signes Française, Editions Rues des Enfants, Slovénie.

Millet, A. (1988) La place de la LSF dans l'intégration scolaire des enfants sourds. Grenoble : Université StendhalGrenoble 3.

Poirot, M. (2013). Les Situations Difficiles Au Travail : Gestion Des Risques Psychosociaux, Elsevier Masson.

Piq, P., Lestienne, C., Sagart, L., Dehaene, G. (2012). La plus belle histoire du langage, Editions du Seuil, Paris.

URL-1. ABC de la langue Française. (n.d.). Langue Française. Consulté le 05 Mars 2020, disponible sur https:// www.languefrancaise.net/Bob/42013

URL-2. Hausser les épaules. (n.d.). Dictionnaire Larousse. Consulté le 06 Mars 2020, disponible sur https://www. larousse.fr/dictionnaires/francais/signe/72700/locution?q= la+langue+des +signes\#11039520

URL-3. Deafness prevention. (2018). World Health Organisation (WHO). Consulté le 07 Mars 2020, disponible sur https://www.who.int/deafness/estimates/en/

URL-4. Language of the World, France. (n.d.). Ethnologue. Consulté le 10 Mars 2020, disponible sur https://www. ethnologue.com/country/FR

URL-5. Quelques chiffres. (n.d.). Fédération Nationale des Sourds de France (FNSF). Consulté le 15 Mars 2020, disponible sur https://www.fnsf.org/

URL-6. Les différents degrés et types de surdité. (n.d.). Wombatoak. Consulté le 18 Mars 2020, disponible sur http://www.wombatoak.com/?page_id=78

URL-7. Histoire de la langue des signes française. (n.d.). La Boîte à Saussure. Consulté le 20 Mars 2020, disponible sur http://laboiteasaussure.fr/lsf_histoire.htm

URL-8. Histoire de la langue des signes française. (n.d.). La Boîte à Saussure. Consulté le 23 Mars 2020, disponible sur http://laboiteasaussure.fr/lsf_histoire.htm

URL-9. La langue des signes française. (n.d.). Wikipedia. Consulté le 23 Mars 2020, disponible sur https:// fr. wikipedia.org/wiki/Langue_des_signes_française

URL-10. La grammaire de la LSF. (n.d.). Consulté le 23 Mars 2020, disponible sur https://sites.google.com/ site/ languesignes/grammaire 
